\title{
LA FORTUNA DE FERNANDO PESSOA EN ITALIA: DEL MITO AL CONOCIMIENTO(1945-1979)*
}

Miguel Ángel Cuevas Universidad de Sevilla

1. Un problema cultural de alcance no pequeño es el del transvase de experiencias literarias de uno a otro contexto nacional y el de su recepción por parte de los eventuales destinatarios; si es cierto que no siempre la disponibilidad del receptor concuerda con la voluntad del emitente, el proceso aún se complica si se tiene en cuenta que ya en su inicio se ha debido producir una alteración del mensaje en el camino que va desde la decodificación del texto original hasta la recodificación de sus constituyentes fónicos y sémicos en otra propuesta textual: es el de la traducción el primer gran escollo que deben salvar los fautores de la transposición; y casi el único, se diría, pues no sólo la materialidad de la palabra importa en un asunto no de exclusiva índole lingüística: antes ha de moverse a través de un entramado de elementos donde juegan tanto la historia de la tradición literaria de la lengua traducida y la de aquella en que se vierte el original, como la peculiar sensibilidad, el tacto intelectual y artístico del traductor. Así, el apelativo nacional que daba pie a estas divagaciones cobra su verdadero sentido: no se apela al viejo concepto heredado de su manipulación tardorromántica, mas a este preciso incardinarse de motivos lingüísticos e históricos. Quienes pretenden salvar el acoso refugiándose bajo el pabellón de las versiones, a pesar del a menudo fructífero estímulo que para su propia obra supongan los puntos de partida, olvidan que no es la recreación sino la traslación en términos de máxima fidelidad y exactitud lo que se exige a una traducción que sea tal.

* Agradezco a la profesora Margherita Spampinato, del departamento de Filología Románica de la Universidad de Catania, la colaboración prestada en los primeros momentos de la búsqueda bibliográfica. 
En el caso particular de italiano, como lengua creada con voluntad de norma que anula, o coloca en situación diglósica, gran cantidad de variantes (a veces casi idiolectales) que se ven relegadas a rango dialectal, el problema deviene más grave: ¿cómo responder a la creatividad lingüística, idiolectal, mediante un código formulado en términos de pureza? Luciana Stegagno Picchio, una de las más responsables portuguesistas italianas, aducía criterios de esta índole con respecto a la dificultad de traducir a Pessoa:

Rispetto a una norma italiana estremamente rigorosa e purista, il portoghese si pone come lingua aperta ad ogni «contaminazione» $[\ldots]^{1}$.

Asimismo Antonio Tabucchi, cuya dedicación a Pessoa ha significado en Italia el final del mito y el inicio definitivo del conocimiento, ha explicitado su conciencia de traductor ante estas cuestiones:

Se una traduzione è, necessariamente, sempre un impoverimento, nel senso che nel passaggio da una lingua a un'altra si verifica una perdita, è inevitabile che anche la nostra traduzione sia in certi casi un impoverimento o una riduzione proprio nell'ambito [...] [della] ambiguità che talvolta, nostro malgrado, è diventata univocità $o$ esplicitazione ${ }^{2}$.

Pero no es sólo a las traducciones adonde cabe acudir para verificar el grado mayor o menor, bien o mal ajustado, de aclimatación de un escritor a unas coordenadas culturales diversas de aquellas en las que ha surgido su obra. Estudios de conjunto o específicos, análisis en profundidad o meras noticias dispersas en cuadros generales, van configurando el contorno en el que se dibujan poco a poco los rasgos que delimitarán su aspecto final. Y quien pretenda historiar estos procesos deberá rastrear desde las alusiones casi indefinidas hasta los más especializados acercamientos profesorales; porque unas y otros, al margen de su hipotética fortuna y objetividad, han contribuido a definir al artista y a su creación. Conviene asimismo, siquiera a título apriorístico, hacer la cuenta con el nivel de influjo real que tienen cierto tipo de publicaciones en el lector medio, aun suponiendo en él un no despreciable interés por las figuras y momentos más caracterizados de las literaturas extranjeras. Quiero decir: se impone reconocer el a veces nulo peso específico en realidades como la que ahora se comenta de revistas cuyo alcance no sobrepasa el ámbito profesional universitario; si es verdad que en ellas se suelen dar los trabajos más acertados, lo es igualmente que -al menos en un primer momento- su capacidad de incidencia es reducidísima fuera de auditorios muy concretos. No es objeto de estas notas abordar arcanas cuestiones de semiología ni de sociología literarias; pero será oportuno no perder de vista preciones como la apuntada. En todo caso, ante la imposibilidad de articular restricciones de teoría de la información sobre un difuso destinatario colectivo, se da cabida aquí tanto a las referencias ligeras como a las recensiones periodísticas o a los artículos de publicaciones especializadas.

1 Luciana Stegagno Picchio, «Pessoa, uno a quattro», Strumenti Critici, 4 (1967), pp. 377-401, p. 392.

2 «Il viaggio fantastico del traduttore» [entrevista a Antonio Tabucchi], Tuttolibri, 12-5-79, p. 14. 
2. Del mito al conocimiento: tal la fortuna de Pessoa en Italia. La extrañeza que provoca el acercamiento a su figura humana ha sido el gran estímulo que ha hecho de sus receptores, embebidos en la dialéctica ambigua de la mistificación, mitólogos antes que escrutadores, parafraseadores antes que exegetas. Mientras su poesía seguía casi desconocida, la referencia Pessoa se iba convirtiendo en lugar común de psicopatología literaria. Primaba el contorno sobre el producto artístico, hasta el punto de constituirse auténticas almonedas de pseudo-psciologismo, toda una parafernalia de esquizoides calamidades en las que campaban a sus anchas Freud y Jung, en tanto que la obra permanecía en el mejor de los casos sólo incompleta y arbitrariamente presentada. Desde que en 1945, en una revista de la recién constituida República Italiana, Armand Guibert, el que sería su traductor francés, daba las primeras noticias sobre el poeta portugués ${ }^{3}$, hasta que en 1979 Antonio Tabucchi publicó el volumen inicial de la única antología orgánica existente hasta la fecha ${ }^{4}$, el camino ha sido largo. Apenas en estos momentos puede afirmarse que el lector italiano tiene acceso a un Pessoa no desfigurado - al menos en la medida en que lo permite la propia plurivocidad de su producción-. Ilustrar las etapas que han debido cumplirse para llegar a la situación actual es el motivo de estas páginas.

3. Parece inevitable ceñirse a criterios cronológicos para avanzar por el camino prefijado; no sólo en base a ellos, sin embargo, hay que hablar de entrada de la «Nota su Fernando Pessoa» (1945) que A. Guibert publicó acompañando las más antiguas traducciones italianas del poeta — cuatro poemas- debidas a Mario Gasparini; pues es una noticia donde se mezclan sin solución de continuidad la puesta en escena del mito y un cierto, si tímido, anuncio de la senda apropiada. El escritor aparece (circunscrito en la tipología simbolista, posromántica, de la indefinición metafórica del paisaje) «[...]per le banchine lungo il Tago fra un'ora e l'altra d'ufficio; e non ci son parole che valgano a cogliere preciso quel che in lui era di fluido, d'elusivo, di ondeggiante, simile alla superficie di quell'oceano sulle cui rive trascorsero i suoi anni $[. ..] \gg^{5}$. Obviamente de pasada, por la brevedad de la nota, se refiere el presentador a continuación a la heteronimia, sin establecer atisbo alguno de búsqueda de organicidad entre las diversas personas poéticas, que son consideradas simulaciones a partir de las cuales cabe plantearse un problema de psicopatología literaria. Pese a lo cual, se puntualiza:

[...] tale fenomeno di frammentazione della personalità, non riesce a dare se non una spiegazione imperfetta $\mathrm{a}$ una ispirazione così multiforme [...]. Senza addentrarci oltre nell'investigazione di uno dei casi più strani di psicopatia letteraria, non ci stupisce trovar nell'opera [di Pessoa] [...] tratti tanto dissimili quali l'espressione positiva e il delirio lirico, l'ellisse e l'eloquenza distesa, lo spirito scientifico e la tendenza al misticismo ${ }^{6}$.

3 Armand Guibert, «Nota su Fernando Pessoa», Poesia, II (1945), pp. 367-369; la nota introdıce la traducción italiana de cuatro poemas (Pobre ceifeira, Natal, Iniciação y Trila na noite) por Mario Gasparini, pp. 370-373.

4 Fernando Pessoa, Una sola moltitudine, a cura di Antonio Tabucchi, Milano, Adelphi, 1979.

5 Guibert, op. cit., p. 367.

6 Ibid., p. 369. 
Si la conclusión de este tópico, y no demasiado fructífero, entreverarse de nociones impresionistas y de sugerencias analíticas no desarrolladas a que nos tiene acostumbrados cierto tipo de crítica de los años centrales del siglo, es por una parte estimulante, cede enseguida a la tentación nunca desmentida de la vaguedad exultante: Pessoa «[...] ha introdotto nella creazione letteraria un'ottica nuova, quella del mistificatore, ossia del «creatore di miti». [...] E su questa nozione dovrebbe essere bassato lo studio che un giorno sarà scritto, quando l'opera s'innalzerà, compatta e multipla, simile a un monumento quadrifronte, in cui la luce e l'ombre alternano i loro giuochi, le loro carezze, i loro segreti» ${ }^{7}$.

El mito, quizá a despecho de Guibert, está servido; porque no es a desvelar la mecánica interna de esa nueva optica adonde dirigirá sus esfuerzos la mayoría de la crítica posterior, sino a la atractiva inmediatez morbosa de la fragmentación de la personalidad.

4. Ya que desvela más de un hábito constructivo de los habitantes de los santuarios de la interpretación, es curioso asistir al nacimiento y al desarrollo de lugares comunes. Uno de ellos, en la exégesis pessoana, es el del super-Camões. Si nos referimos a su recepción en Italia, el proceso se amplía y se restringe a un tiempo: pues se admite como mero dato situacional sin el mínimo posicionamiento crítico y, además, se anulan las referencias que lo contextualizan. Giuseppe Carlo Rossi, en su Storia della letteratura portoghese (1953), lo anuncia como si de un elemento consabido se tratara: no falta en Portugal quien equipara la poesía de Pessoa $\gg[\ldots]$ a quella delle più grandi figure nazionali, e addirittura del Camões $[. ..] \gg^{8}$. Sin más. P. A. Jannini, en la antología Pagine della letteratura portoghese (1955) (en la que incluye seis poemas de Pessoa, tres del Ortónino y uno de cada heterónimo principal), adorna la mención:

Pessoa,per la sua singolare personalità e la profunda ispirazione legata alle radici stesse dell'anima lusitana -si è persino parlato di un genio pari a quello di Camões - s'impone come uno dei piú grandi poeti della letteratura portoghese di tutti i tempi ${ }^{9}$.

Al margen de lo cual, el antólogo hace honor al mito cuando nos cuenta del atormentado poeta mesiánico, nostálgico de ensoñaciones y exaltador de patriotismos, del sebastianista Pessoa de Mensagem.

El delicado encanto del tópico, en la versión de Jannini, se aja cuando el lector interesado conoce por mediación de Tabucchi sus circunstancialidades originarias: la historia de super-Camões como un eco de los artículos que el Pessoa aprogramático, francotirador de conciencias literarias bienpensantes, publicara

7 Ibíd., Ibídem.

8 Giuseppe Carlo Rossi, Storia della letteratura portoghese, Firenze, Sansoni, 1953, pp. 293 s., p. 293.

9 Pagine della letteratura portoghese, a cura di P.A. Jannini, Milano, Nuova Accademia, 1955, p. 373; en pp. 384-393 se incluye la traducción de seis poemas junto a sus originales portugueses, discriminando la autoría de los mismos: Pobre ciefeira, Dizem?, Liberdade, de Pessoa; un Poema, de Caeiro; Pecado original, de Campos; una Ode, de Reis. 
en $A$ Aguia en 1912, un discurso egotista bajo la apariencia de propuesta y augurio de renovación de la poesía portuguesa ${ }^{10}$.

5. Se sabe que los manuales de historia literaria suelen ser catálogos de simplificaciones. No escapa a la norma el de Rossi, pero en él se leen cosas de interés: se habla de una «[...] personalità umana e artistica complessa e sconcertante [la quale assume] un eccentrico atteggiamento artisticamente significativo [... ${ }^{11} ; \mathrm{y}$ se caracteriza la obra de Pessoa en términos que, si bien no sobrepasan el marco de la perífrasis explicativa de la plurivocicad de los heterónimos, no se esperarían en una tan rapidísima ojeada como la que por otros conceptos resulta evidente:

Nel complesso, la caratteristica di tale opera poetica si può additare in una sconcertante altalena, fra i compiacimenti del raziocinio e gli abbandoni all'emozione, dove pensiero e sentimento si «calano» in un linguaggio difficile e che allo stesso tempo si palesa attraente dominatore dei mezzi verbali, in una «costante» $\mathrm{e}$ in un equilibrio ignoti da tempo alla lirica portoghese ${ }^{12}$.

Son sugerencias estas que hacen pensar en el análisis como elemental consecuencia en la ocasión propicia. Y así parece suceder cuando en La civiltà portoghese. Profilo storico e storico letterario (1975) Rossi dice considerar la heteronimia como «[...] trasferimento di una singolarissima lucidità mentale al mondo della poesia», palabras que semejan sintética respuesta a la abstrusa pretensión de interpretación cuasi-psicologista de Luigi Panarese, autor de la primera antología italiana de Pessoa. Pero, en lugar del detenimiento, sucede la simplificación: «È una poesia gioco e problema filosófico allo stesso tempo». en todo caso, desde las frases iniciales de esta nueva presentación, se perfila la ratificación del mito, la marginalidad, la diferencia, el golpear de la desgracia sobre el Pessoa niño: «[...] di ascendenza di cristianinuovi, nipote di una nonna paterna pazza, rimasto orfano dal padre a cinque anni $[. ..] \gg{ }^{13}$. El reclamo biográfico sigue su curso; la escritura,si acaso, en tanto que trasunto de la vida.

6. En otro manual, La letteratura portoghese (1961), de Francesco Piccolo, se halla una valoración independiente, que escapa a la senda trillada del mito y huye del recurso al perfil humano como justificación enaltecedora de la arquitectura poética: la obra, antes que otra cosa, es indicio de la falta de cohesión interna, de equilibrio, del espíritu de Pessoa.

[...] l'opera di Pessoa è la testimonianza di una ricerca di un punto di appoggio da parte del poeta che vuol chiarire i motivi del sovrapporsi continuo nel suo spirito di intuizioni delle quali una sintesi non è mai raggiunta. Sicché Fernando Pessoa rimane come una personalità sdoppiata in multiformi aspetti dei quali l'uno non si riconosce nell'altro: un insieme, insomma, di personalità incompiu-

10 Tabucchi, «Interpretazione dell'eteronimia di Fernando Pessoa», Studi Mediolatini e volgari, XXIII (1975), pp. 139-187, p. 161.

11 Rossi, op. cit., p. 293.

12 Ibid., p. 294.

13 Todas las referencias en Rossi, La civiltà portoghese. Profilo storico e storico letterario., Milano, Mursia, 1975, pp. 189-192. Del mismo autor puede asimismo verse «Il caso poetico Fernando Pessoa», Quaderni Iberoamericani, 3 (1953). 
te, di poca vitalità ciascuna, ciascuna apparsa nel calendario della vita e subito cancellata, scacciata dalla successiva. Che Pessoa abbia conoscenza di questo difetto di sintesi e di unità del suo essere, che sia stato cioè al corrente della propria disgregazione interiore è fin troppo chiaro dal senso di triste, melanconica ironia della sua arte che dichiara il carattere cosciente-incosciente del mondo che vi è celebrato ${ }^{14}$.

Se trata casi de una reducción al absurdo de la vaga atracción provocada por el fingidor; sin duda Piccolo no comprendió a Pessoa, desde una concepción cimentada en una noción de arte que fueron bombardeando todas y cada una de las vanguardias históricas; ni supo ver, ni intuir, la organicidad de un sistema creativo en que las partes se suceden en la configuración de la obra heterocósmica, como L. S. Picchio o el ubicuo Tabucchi ${ }^{15}$ se han encargado de mostrar. No colabora, pues, esta referencia al conocimiento del poeta, a su buena fortuna en Italia; pero, aun desde el error, es expresión propia, significativa, no mera reproducción de la tópica sancionada.

7. Hasta la aparición de Poesie, di Fernando Pessoa (1967), reeditadas como Imminenza dell'ignoto (1972), la antología de Luigi Panarese, el público italiano contaba sólo con los poquísimos poemas traducidos por Gasparini y Jannini ${ }^{16}$. Pero lo que podría haber sido el inicio del conocimiento no fue sino crisol del mito, de la distorsión, de la incomprensión en suma: en efecto, en una «Cronistoria della vita e delle opere» del poeta se aglutinan y cobran aspecto definitivo todas las sugerencias de interpretación extraliteraria de la crítica precedente. De Pessoa parece interesar exclusivamente la «[...] morbosa sensibilita [la quale] ricaviamo da innumerevoli testimoni $[\ldots] \gg^{17}$ : así, se resalta la presencia del complejo edípico, se ilustran sin la menor concesión a la ironía (es decir, a la duda como sistema epistemológico) sus mortificantes autodiagnosis neuróticas..., para concluir en un tratado de los heterónimos explicado por via jungiana en el que Pessoa aparece como un nuevo Prometeo:

[...] intuisce quale può essere la via della salvezza e la imbocca: accetta di essere diverso dagli altri, ammette che la vita altrui è la normale, ma abdica ad essa e si uniforma alla propria, rimanendone «soddisfatto». Ormai vive libero dalla preoccupazione di piacere e di imporsi agli altri; si vuole com'è; sa di essere un genio e che gli si impone una missione divina. Perciò non si considera più male-

14 Francesco Piccolo, La letteratura portophese; cito por la segunda edición, aumentada, de la obra, Firenze-Milano, Sansoni-Accademia, 1970, p. 300.

$15 C f$., respectivamente, «Pessoa, uno e quattro» e «Interpretazione dell'eteronimia di Fernando Pessoa».

16 Al margen de lo cual, L. Frediani preparó un ensayo introductivo y tradujo una breve selección de poemas de Pessoa en Estudos italianos en Portugal (Lisboa), 14-15 (1955-56), pp. 145-168; pero es algo que queda fuera del alcance del lector italiano, como lo es asimismo la «Nota introductoria» a la traducción italiana de la Ode maritima que publicó Orietta del Bene en el volumen correspondiente a los números 33-34-35 (1970-71-72) de la misma revista. Por otro lado, el propio Panarese había dado «Tre sonetti di Fernando Pessoa» (II, V y X de Passos da Cruz) en Quartiere, n. 3, s.a.

17 Fernando Pessoa, Imminenza dell'ignoto, studio e antologia poetica a cura di Luigi Panarese, Milano, Accademia, 1972, p. 7. En la primera edición (Milano, Lerici, 1967) se incluía una desmesurada «Cronistoria della vita e delle opere» del poeta que aquí aparece reducida a cauces menos desorbitados, si bien aún confusos y sumamente discutibles. 
detto, ma eletto, non ha bisogno di apparire quello che non è, gli basta essere chi è, cioè un poeta, un creatore, un essere superiore, aristocratico, che disprezza la celebrità perché è una grossolanità indegna di anime delicate e che si paga con la perdita della vita intima offerta alla curiosità indiscreta di tutti ${ }^{18}$.

Tal es el tono que domina en la introducción de Panarese, a mitad de camino entre el metalenguaje pseudo-científico y la altisonancia del genio elegido, demiurgo. Por lo demás, la disertación sobre los heterónimos no es sino paráfrasis de las afirmaciones entre ellos cruzadas, sin huella de interpretación sistemática. Lo más que se alcanza es la simplista afirmación de que «Si è sempre riconosciuto a Pessoa un certo spirito mistificatore («simulazione»), una certa ironia, una tendenza allo scherzo, tuttavia il fenomeno dell'origine degli eteronimi non ci sembra puro frutto di fantasia surrealista, ma elaborazione letteraria di un'esperienza subreale dolorosamente sofferta» ${ }^{19}$.

La afirmación de la función mesiánica del poeta concluye, sintomáticamente, en un errado concepto de la herencia romántica; la obra de Pessoa «[...] acquista un preciso significato ontologico e gnoseologico [portare «a compimento una missione a favore della umanità con i mezzi messigli a disposizione»], conforme all'idea romantica della poesia da cui discende Pessoa di generazione in generazione [...] $\gg^{20}$. Mario Luzi, en una breve recensión de la antología (1967), pone el dedo en la llaga por lo que toca a estos extremos, al proponer la creación de los heterónimos - fuera de un mero juego de alternancia de voces- «[...] come un aspetto molto singolare che prese la crisi della soggettività nella creazione posteriore al romanticismo ${ }^{21}$. Estamos, pues, ante dos captaciones diversas de la tradición romántica: la de Panarese elemental, gruesa, al cabo falaz; la de Luzi en la vía justa. Porque la afirmación del yo en los mejores ejemplos de la poesía romántica no fue sino índice inequívoco de la pérdida de unicidad, de la dispersión, de la búsqueda consciente y apasionada —las más de las veces infructuosadel equilibrio en la naturaleza; y es esa herencia, con su concepción de lenguaje simbólico, la que desgajada por la realidad de la indagación de una armonía imposible, subyace en Pessoa como en todo el arte contemporáneo. El leve escalpelo del poeta Mario Luzi ha acertado en la disección, mientras todo el atrezzo psicologista de Panarese fomenta el mito de las morbosas disfunciones de un Pessoa intimista y un tanto decadente en su sebastianismo redivivo. ¿Dónde queda, en fin, el acercamiento analítico en términos de literaturidad? No existe. El mito se presenta, ahora, ya definitivamente condimentado. Pues, además, como apunta Tabucchi, el poeta propuesto por el antólogo está deformado, incompleto: «[...] è privilegiato il Pessoa intimista e crepuscolare del Cancioneiro e della Quadras

18 Panarese, «Introduzione biografico-critica», Imminenza dell'ignoto, pp. $23 \mathrm{~s}$.

19 Ibíd., p. 32.

20 Ibid., p. 24.

21 Mario Luzi, «Il misterioso Dom Fernando», La fiera letteraria, XLII, no. 29, 20-7-1967, p. 21. Vid., asimismo, la nota de R. Jacobbi en Paese-Libri, suplemento de Paese-Sera, 19-5-1967. 
ao Gosto Popular, e il messianico-nazionalista di Mensagem; mancano i testi fondamentali di Campos e di Caeiro [...]» ${ }^{22}$.

8. En la recensión de Luzi hay una proposición que, más de veinte años después, enlaza con la inicial de Guibert: Pessoa «Si esprime [...] per alienazzioni successive, divergenti, che tuttavia mantengono un nesso organico più che dialettico con la sua parte consapevole e volontorai» ${ }^{23}$. La sugerencia, dadas las limitaciones de espacio obligadas por el medio, no es desarrollada por el propio Luzi; pero creo oportuno destacarla, pues por aquí va a dirigir sus pasos la crítica más capaz a la hora de desvelar el mundo literario creado por el poeta, más allá de oscuras pretensiones de análisis de una personalidad atormentada.

Así, Luciana Stegagno Picchio, en el que quizá pueda considerarse como el primer estudio serio de aproximación al problema de la heteronimia publicado en Italia, «Pessoa, uno e quattro» (1967), inicia la tarea, en palabras de Tabucchi, de «[...] strappa[re] Pessoa dal suo multiplo inferno di solitudine culturale nel quale lo ha confinato tutta una critica abbagliata dal suo «caso», per leggerlo, finalmente, in un contesto culturale europeo a lui coevo [...]» ${ }^{24}$. El trabajo es asimismo un comentario a la antología de Panarese, de la que se dice, en síntesis: «[...] un'antologia è un'interpretazione e l'antologia di Panarese [...] finisce col dare di Pessoa un'immagine sfocata, parziale» ${ }^{25}$. Al comentar la presentación de los textos se hacen afirmaciones de verdadero alcance:

È sempre assurda e opinabile la storia del non-esistito. Ma quanto sapiamo dei suoi programmi ci fa sicuri che Pessoa vivo non avrebbe pubblicato i suoi versi como ci vengono ora proposti, cosí romanticamente sciolti, cosí impressionisticamente testimoni dell'attimo creativo. Li avrebbe chiusi nella struttura di un canzoniere pieno di trovate forse [...], ma rivelatore unitario della sua poliedricità. [...] E il caso Fernando Pessoa avrebbe potuto essere studiato al di fuori dell'attuale ipertrofica mistificazione di un gioco letterario, al di là di un biografismo, fittizio, ma non perciò più essenziale: essere studiato cioè in chiave di letteratura più che di scandalismo letterario ${ }^{26}$.

Búsqueda de la literaturidad: he aquí la llave maestra de la interpretación, que sólo desde este momento comenzará a abrir las puertas del conocimiento, a sellar los conspicuos pasadizos del mito. La Picchio, explícitamente, no va de momento más allá —sí lo hará en ulteriores aportaciones-: «Il problema non era di entrare nel gioco dell'eteronimia, ma di dichiarare la volontà di struttura, di organizzazione di Pessoa» ${ }^{27}$. Bastante es, sin duda, inaugurar una vía que había permanecido demasiado tiempo intacta.

22 Tabucchi, «Fernando Pessoa. Baedeker bibliográfico», Quaderni portoghesi, 2 (1977), pp. 195-204, p. 204.

23 Luzi, op. cit.

24 Tabucchi, «Fernando Pessoa. Baedeker bibliográfico», p. 198.

25 Luciana Stegagno Picchio, op. cit., p. 377.

26 Ibid., p. 379 s.

27 Ibíd., p. 386. Luciana S. Picchio es autora además de trabajos específicos que por diversas razones —el ámbito de su publicación y su propia especificidad — no son para comentados aquí: «Les 
9. Las ingenuidades también cuentan en este juego de metalenguajes de la crítica: sus sencillos materiales, sin embargo, no cuadran con la magneficiencia del monumento construido al mito y son demasiado frágiles para contribuir a un conocimiento apenas cimentado. Orietta del Bene sigue la pista de la relación animista entre el maestro Caeiro y su extraño pupilo Pessoa: «Alberto Caeiro: un atteggiamento di Fernando Pessoa» (1968). La poesía de Caeiro, dice a modo de caracterización genérica, «[...] possiede l'incanto sottile, e pur non privo di misteriosa severità, di certi paesaggi montani del Portogallo [...]» ${ }^{28}$. Mas donde la simplicidad (vale decir: la absoluta carencia de comprensión de la organicidad del sistema heteronímico, entre otras cosas) alcanza sus máximas cotas es a propósito de las composiciones del Pastor Amoroso, que «[...] hanno una semplicità singolare, una così innocente freschezza che il lettore è portato a immaginarle uscite non dalla penna di Fernando Pessoa [...] ma dalla bocca ingenua di Adamo che si accorge di Eva. Presto però l'illusione sfuma e il poeta-pastore si avvede che la donna lo allontanava della Natura perchè dava alla sua esistenza un carattere troppo soggettivo: dal che è ragionevole dedurre che Fernando Pessoa ipse, solitario e misogino, [...] l'ha vinta sul «mestre Caeiro», panteista innamorato» ${ }^{29}$.

Basta como muestra.

10. El más beligerante activista contra el mito de la fragmentación humana de Pessoa como justificación ubicua y engrandecedora de su actividad poética es Giuseppe Tavani, quien en su antología De Pessoa a Oliveira (1973) insiste en hacer caso omiso de la discriminación entre los heterónimos y el Ortónimo, lo que considera «[...] impensabile e criticamente assurdo lavoro [...] $»^{30}$. Y continúa:

[...] l'insistenza del poeta sull'autonomia dei tre personaggi che egli veniva creando, non può non convalidare il sospetto di una mistificazione non più strumentale e riservata a se stesso ma ora gratuita e riservata agli altri ${ }^{31}$.

Es una dirección la de Tavani que no ha tenido seguidores en la especulación italiana, antes la contestación tajante, contrastada de Tabucchi, a quien es justo considerar uno de los mejores intérpretes de Pessoa. Con todo, es interesante, al menos en sus consecuencias últimas; por ello merece ser observada con detalle:

Pessoa [...] aveva l'ambizioso progetto (sminuzzato in una serie di «sottoprogetti», tutti irrealizzati) di costruire al proprio genio il monumento piú vistoso: un

oxymores dialectiques de Fernando Pessoa» [en colaboración con Roman Jakobson], Langages, 12 (1968), pp. 9-27, y «Marinetti et le Futurisme mental des portugais», Atti del Colloque Marinetti, París, 1976.

28 Orietta del Bene, «Alberto Caeiro: un atteggiamento di Fernando Pessoa», Annali del Istituto Universitario Orientale di Napoli (Sezione Romanza), X, I (1968), pp. 153-168, p. 164.

29 Ibíd., p. 159. Otros dos estudios que se publican en estos años, cuyo alcance no modifica substancialmente la línea aproximativa hasta ahora trazada, son los de Marilia Bonicontro, «La presenza di Orazio nella poesia di Riccardo Reis, eteronimo di Fernando Pessoa», Pensiero e scuola, 4 (1986), y Raffaella Longobardi, «Fernando Pessoa: ancora sugli eteronimi», Annali del Istituto Universitario Orientale di Napoli (Sezione Romanza), XII, I (1970), pp. 43-90.

30 Da Pessoa a Oliveira. La moderna poesia portoghesa. Modernismo. Surrealismo. Neorealismo, a cura di Giuseppe Tavani, Milano, Accademia, 1973, p. 9.

31 Ibid., pp. $10 \mathrm{~s}$. 
«canzoniere» meticolosamente e puntigliosamente structturato, nel quale la sua personalità si distribuisse ordinatamente tra quattro poeti (tre eteronimi e l'ortonimo). Ciascuno degli eteronimi avrebbe dovuto costituire la presa di coscienza di una data realtà da un certo punto di vista, avrebbe dovuto cioè consentirgli di affrontarsi e confrontarsi, di contemplarsi in altrettanti specchi deformanti per poi ricostituirsi e riconoscersi compiutamente nell'ortonimo, summa e unica verità della sua esperienza esistenziale. L'operazione, intrapresa probabilmente in piena sincerità di intenti per far fronte all'angoscia di una condizione umana dilacerata [...], dovette diluirglisi tra le mani, apparire sempre più come un gioco, banale e un po'infantile, che aveva adempiuto alla propria funzione catalizzatrice e sperimentale ma che non si offriva ormai piú come valido criterio strutturale del monumento a se stesso quale egli l'aveva confusamente concepito ${ }^{32}$.

La incomprensión del mundo pessoano, del otro mundo en sí que es su obra, se resalta aún si se tiene en cuenta que, en opinión del crítico, el hecho de que los personajes de la heteronimia menor (Bernardo Soares, Coelho Pacheco,...) no aparezcan tan marcadamente delineados como Caeiro, Reis o Campos en el arca del poeta «[...] conferma, tuttavia, come la sincera ricerca di sé intrapresa con la creazione dei primi eteronimi, si fosse man mano ampliata oltre ogni misura, diluendosi in semplice gioco pseudonimico» ${ }^{33}$.

Hablaba antes, sin embargo, del relativo interés del precipitado de este antimitólogo combativo: de la ficha que acompaña los poemas que traduce resulta una imagen de Pessoa en base a su biografía externa, a su actividad de militante fundador de revistas vanguardistas, a las fechas de publicación de su obra; ni un solo desmelenamiento psicologista. Un no rotundo al mito.

Además de la oxigenación que este nuevo aire proporciona, es de valorar la elección que hace Tavani de los textos de Pessoa, entre los que da los emblemáticos Ode maritima y Tabacaria, no incluidos en la antología de Panarese, que se va cada vez más perfilando como insuficiente.

11. Llegamos a Antonio Tabucchi o, lo que es lo mismo, al ocaso del mito, al conocimiento ajustado a realidad de Pessoa en Italia. Tabucchi es autor de un magnífico ensayo de intelección del sistema heteronímico en cuanto tal: «Interpretazione dell'eteronimia di Fernando Pessoa» (1975). Más que una referencia detallada de las conclusiones a que llega, interesa aquí pararse en los presupuestos desde los que articula su aproximación al poeta, pues significan de una vez la afirmación de la necesidad de un discurrir interpretativo en los antípodas de una tradición decimonónica de identificación entre trayectoria poética y devenir vital: será la única manera de dirigirse al fenómeno literario sin los deslumbramientos de un biografismo más o menos agudo, pero casi siempre morboso, y fútil desde el punto de vista crítico.

Pessoa è uno di quei labirinti in cui, per dirla con parole sue, «ogni strada porta da ogni parte» e «ogni punto è il centro dell'infinito». E di tali labirinti esistono

32 Ibid., pp. $8 \mathrm{~s}$.

33 Ibíd., p. 8 n. Tavani es además autor de otros dos trabajos que sobrepasan el ámbito de estas notas: «A propósito duma antología», Coloquio / Letras (Lisboa), no 25 (1975), y «Análise Ritmética de un Poema de Fernando Pessoa», Estudos italianos en Portugal, 38-39 (1975-76). 
infinite letture, tutte ugualmente legittime. Ma intendiamoci: ogni interpretazione è legittima purché sia a posteriori; perché finora i giudizi aprioristici cui Pessoa è stato sottoposto appaiono più supposizioni che deduzioni legittime. Non si può dire chi è Pessoa senza sapere che cosa significa la poesia di Pessoa, e non si può dire che cosa significa la poesia di Pessoa senza conoscerne il funzionamento. / [...] Tutti i corridoi poetici pessoiani sono incrostati dai sedimenti di quel luogo comune che vuole la «vita» quale causa o motivazione dell'opera. Rifiuteremo di ascoltare le suadenti diagnosi che insinuano complessi edipici, traumi infantili e disfunzioni sessuali per la decifrazione della molteplicitá eteronimica. Quell'assioma intollerabile e astuto che voule la «vita» prima della opera, addirittura occasione o movente della seconda, mai fu tanto sterile, in termini critici, come nel caso di Pessoa $[\ldots]^{34}$.

Cogido el testigo del relevo de manos de la Picchio, va a ser el de Tabucchi el primer intento sistemático para captar la organicidad del orbe heteronímico creado por el poeta portugués: "In realtà i personaggi non sono mobili, ma occupano un punto fisso. Sono, cioè, funzioni $[. ..]{ }^{35}$. Así - muy sintéticamente, puesto que el interés de estas notas no es tanto desvelar las conclusiones cuanto historiar un proceso-, el Ortónimo, para creer en el mundo, hace que el maestro Caeiro lo construya tal como ya es; Caeiro levanta una ficción sobre la ficción: el universo que Reis acepta y del que Campos se hace dueño, desarrolla y agota, obligando al Ortónimo a que postule de nuevo a Caeiro; punto de partida y final de la jornada se enlazan, y se abre la puerta a la apreciación del mesianismo irónico de Mensagem: un mundo de ficción creado sobre una realidad ficticia a través de la ficción de la poesía: «[...] la costruzione è un circolo infinito da ricominciare infinite volte» ${ }^{36}$.

En un trabajo posterior, el propio Tabucchi enjuicia en clave de ligera agudeza esta artículo: «Resta al lettore la consolazione di non sentire più parlare delle inflazionate banconote psicoanalitiche che per l'eteronimia hanno ormai perso valore d'acquisto» ${ }^{37}$. Mas su interpretación de la heteronimia no puede desgajarse de aportaciones ulteriores. En «Pessoa o del Novecento» (1977) postula el abandono para con el poeta portugués de la bizarría de la originalidad exclusiva: se trata de un estimulante ensayo de análisis de la descomposición de la conciencia como característica de la literatura de siglo XX, en el que se rozan los míticos temas de la genialidad y la locura del escritor, reducidos a términos literarios:

[...] la follia circola anche nell'opera. E non tanto per quei tuffi nell'esoterismo, per l'ermetismo quasi nigromantico di certe poesie dell'Ortonimo; ma proprio per il fatto esterno, per la costruzione, per l'impalcatura di un'opera che si regge su personaggi così diversi, così autonomi, così contrastanti: il neoclassico Reis, il «naif» Caeiro, l'avanguardista Campos e l'introspezionista Ortonimo. [...] C'è

34 Tabucchi, «Interpretazione dell'eteronimia di Fernando Pessoa», pp. 141 s.

35 Ibid., p. 185.

36 Ibíd., p. 138. Antes de este trabajo definitivo, Tabucchi había publicado una pequeña nota, «Pessoa è un esempio di antiteatro o di «teatro statico», Il Dramma, 8 (1970). En Portugal además ha salido «Una charada de Fernando Pessoa», Estudos italianos en Portugal, 38-39 (1975-76).

37 Tabucchi, «Fernando Pessoa. Baedeker bibliográfico», p. 199. 
odore di follia, indubbiamente. Follia che però a sua volta non risolve nessun problema. Non risolve nessun problema perché, nella sua realizzazione, l'eteronimia «funziona» alla perfezione: se cioè lascia perplesso il meccanismo che può provocare la dissociazione, ogni «dissociato» è un poeta autentico, autosufficiente, perfetto. La follia è dunque estrinseca all'opera: intrinsecamente è ingabbiata, razionalizzata, risolta. / E a questo punto il discorso si sposta necessariamente sul piano della letteratura, perché forse non c'è altra legittima maniera di impostarlo. Pessoa, con la sua psicopatologia [...] si ritira discretamente fra le quinte della sua scenografia e ci consegna l'opera, ci invita alla letteratura ${ }^{38}$.

El escritor queda precisamente configurado, en la intensidad de su laberinto epistemológico, como un poeta del conocimiento:

[...] riduciamo il problema all'aspetto letterario. Cerchiamo cioè di capire in cosa consiste, letterariamente, il fatto eteronimico di Fernando Pessoa. / L'operazione di Pessoa consiste nel tradurre in un fatto così clamoroso e per certi aspetti persino istrionesco come quello della creazione eteronimica, l'elemento più rivoluzionario del Novecento: la Coscienza ${ }^{39}$.

El tema, que fue apuntado por Luzi en implícita oposición a la barahúnda de Panarese, se desarrolla coherente hasta sus últimas consecuencias en «Un baule pieno di gente» (1979), la introducción a Una sola moltitudine, antología que signa el grado de madurez de la captación de Pessoa en Italia:

Con Pessoa una delle grandi preoccupazioni della letteratura della nostra epoca, l'Io, entra in scena e comincia a parlare di se, comincia a riflettere su di se. Attraverso un'impostazione meticolosa, da referto psicoanalitico, l'eteronimia non è altro che la vistosa traduzione in letteratura di tutti quegli uomini che un uomo intelligente e lucido sospetta di essere. [...] Ma è evidente che Pessoa, con l'accurata stesura dei diversi copioni attribuiti a ogni suo altro, opera non tanto nella direzione verticale dell'irresponsabilità del creatore, tipica delle poetiche tardo romantiche [...] quanto nella direzione del creatore che si fa responsabile e dominatore di un atto inizialmente irresponsabile, e che si muove sul piano orizzontale di un sistema. [...] L'eteronimia o dell'uomo multiplo;[...] l'Io è uno sguardo in dentro, e solo in questa direzione: il microcosmo diventa macrocosmo, il soggetto esclude l'oggetto, anzi il soggetto diventa oggetto di se stesso, si pone a se stesso come altro da sé. Non c'è più l'altro, ma l'alter ego: l'eteronimo ${ }^{40}$.

Detallada a continuación la nómina de los heternónimos, desde los más caracterizados hasta aquellos que lo son en menor grado, de sus complejidades, de sus mutuas relaciones, de la magnitud e intensidad en fin de la galaxia pessoana, concluye, en abierta contradicción con los juicios de Tavani: «A questo punto,

38 Tabucchi, «Pessoa o del Novecento», Quaderni portoghesi. I (1977), pp. 17-36, pp. 24 s.

39 Ibid., p. 25.

40 Tabucchi, «Un baule pieno di gente», [introducción a] Fernando Pessoa, Una sola motltitudine, pp. 11-35, pp. 25 s. Esta introducción supone en amplia medida el conjunto reescrito - simplificando a veces, ampliando otras- de «Interpretazione dell'eteronimia...» y de «Pessoa o del Novecento». 
senza dubbio, Pessoa è l'eteronimia: parlare semplicemente d'artificio letterario sarebbe sufficienza o presunzione» ${ }^{41}$.

12. Desvanecida la niebla espesa del mito que difuminaba el contorno del escritor y lo hacía aparecer de mágicas dimensiones, de múltiples transparencias, es ahora el momento del análisis detenido, del juicio de pormenor, de la construcción progresiva de un conocimiento vario pero siempre decididamente atento a delimitar el perfil del poeta Pessoa, del creador. La revista Quaderni portoghesi sale a la luz en Pisa (1977) con dos numeros dedicados a su figura: la ratificación, la señal de un interés ya suscitado que provoca incluso lecturas externas. Es una colección de artículos, en su mayoría de especialistas italianos y portugueses, por lo general de un alcance muy particular cuya propia restricción les confiere por lo común considerable altura ${ }^{42}$. Conviene destacar — no debido a su mayor valor intrínseco en relación con las demás aportaciones, sino por lo que tiene de índice de una actitud genérica que se va imponiendo- la entrevista que el pertinaz Tabucchi realiza al poeta Andrea Zanzotto. De nuevo en la línea de la sugerencia formulada por Luzi, comenta Zanzotto el hecho de la heteronimia:

Questo io che produce suoi duplicati [...] e che finisce per non sapere più se sia l'«originale» o una copia, [...] dichiara il fallimento di un discorso in cui un soggettivismo assoluto non puó che esplicitare la propria irrealtà $[\ldots]^{43}$.

El tema de la hipotética unicidad de Pessoa en la organicidad de un sistema de ubicación de las distintas voces que asume, es preocupación sabida de Tabucchi, ante cuyas preguntas responde el entrevistado:

Pessoa resta un unicum perchè esiste in lui una inestricabilità tra il sentimento dell'eteronimo come fatto letterario e la possibile tragedia di una realtà psicologica scissa che ne fa da supporto, in una zona di ambiguitá in cui la spaccatura

41 Tabucchi, «Un baile pieno di gente», p. 33.

$42 \mathrm{Al}$ margen de los artículos que, por su alcance más general en el objetivo analizado, se comentan en estas notas, la nómina de los trabajos que aparecen en Quaderni portoghesi es la siguiente: I: Jacinto do Prado Coelho, «Qualcosa di nuovo su Antonio Mora», pp. 37-43; Cesare Segre, «Il sogno del sogno in una poesia di Pessoa», pp. 45-54 [aproximación semiológica a Ó naus felizes, del Ortónimo]; Stephen Reckert, «Fortuna e metamorfosi di un «topos» nella poesia di Pessoa», pp. 55-94 [análisis y desarrollo del tema de la «bella desconocida»]; Armando Martins Janeira, «Zen nella poesia di Pessoa», pp. 95-116; María José de Lancastre, «Peregrinatio ad loca fernandina: la Lisbona di Pessoa», pp. 117-135; "Jorge de Sena risponde a tre domande su Pessoa», pp, 137-158 [entrevista de Luciana Stegagno Picchio sobre los poemas ingleses de Pessoa como clave para la interpretación de la poesía portuguesa. De Jorge de Sena se había publicado en Italia «Quattro poesie antisalazariste di F. Pessoa», Carte segrete, 26 (1974), traducción del trabajo homónimo aparecido en Portugal en Seara Nova, n. 1545 (1974), pp. 19 s.]. II: Almeida Faria, «Pessoa che pensa Campos che sente», pp. 11-25 [crónica de una polémica sobre estética]; Luciana Stegagno Picchio, «Chuva oblíqua: dall’infinito turbolento di F. Pessoa all'Intersezionismo portoghese», pp. 27-63; Y. K. Centeno, «Episódios / A Múmia di Pessoa: un testo chiave per lo studio dell'ermetismo in Fernando Pessoa», pp. 65-101; Alberto Pimenta, "O Último Sortilégio di F. Pessoa», pp. 103-126; Silvano Peloso, «L'ultima maschera di Fernando Pessoa: le Quadras ao gosto popular», pp. 127-147; Manuel Poppe, «Montaggio di prose: l'enigmista», pp. 149-153 [collage a partir de las páginas íntimas y de autointerpretación del poeta]; Eduardo Lourenço, «Walt Whitman e Pessoa», pp. 155-184.

43 «Andrea Zanzotto risponde a tre domande su Pessoa» [entrevista de Antonio Tabucchi], Quaderni portoghesi, 2 (1977), pp. 185-194, p. 193. 
della persona-Pessoa [...] genera altri io dall'aspetto di minotauri che stanno tra l'allucinatorio e il più spericolato gioco letterario ${ }^{44}$.

Mas todo ese laberinto es sin duda difícilmente reconducible a unidad desde una óptica de identificación del nombre y la realidad nombrada:

Nell'enigma di Pessoa vediamo [...]numerosi personaggi paralleli, relativamente minori degli altri eteronimi più consacrati, affacciarci in continuazione e sovrapporsi, accompagnarsi ai primi. Si trata di una folla di potenzialità che tenderebbe a minare la vita / realtà intesa come un puzzle in cui ogni pezzo può avere un nome, senza che possa costituirsi il nome del «tutto», se non a barlumi vaghi nella precarietà di un ortonimo «portante», anche se non privilegiato. Il «tutto» è uguale a ciascuna sua parte. Una realtà non ordinabile, nè reconducibile ad unità [...]. Si pensi all'oscura angoscia ma anche alla sottile perfidia del non volere né poter chiamare pseudonimi questi segni. Perché, di fatto, sembra che tutto venga a giocarsi proprio intorno al nome, ai nomi. In Pessoa un io diviso, che non può nemmeno sospettare lo pseudonimo, va a bracetto con un superlogico «fingidor» ${ }^{45}$.

Sólo a partir de la comprensión del tejido de literaturidad en el que es obligado ubicar el empeño de Pessoa será posible recomponer una relativa, virtual, homogeneidad:

Sarà [...] una capillare analisi delle strutture fonicoritmiche, sintattiche, sèmiche, dei loro intrecci di sovrapponibilità, opposizione, contestualità, a dirci «il profondo» dell'opera di Campos, Reis, Caeiro ecc., in modo di assicurarci veramente che essi siano qualche cosa di più che nomina e, in definitiva, flatus vocis, nonostante i loro conclamati profili e «programmi» tanto differenti. Insomma, la ricerca effettuata sui tessuti soprattutto dei componimenti poetici farebbe probabilmente constatare un superamento delle barres nominali, portando a ritrovare un dato omogeneo [... $]^{46}$.

El hincapié, pues, por fortuna, se hace ya sobre el universo creado.

13. Una traducción orgánica, tendente a desvelar la totalidad de la obra de Fernando Pessoa, no detenida en la envoltura de mera autoconfesión de algunos fragmentos ni mutilada por el carácter cerrado que la sanción de cierta crítica ha otorgado a otros, seguía sin aparecer en Italia. En su «Baedeker bibliográfico» (1977) Antonio Tabucchi anunciaba la próxima publicación de una nueva antología a su cuidado. Así con Una sola moltitudine (1979; un segundo volumen ha salido en 1983) se cumple el ciclo que se inició treinta y cuatro años atrás con las primeras noticias que daba Armand Guibert: existen los textos -en traducciones de Rita Desti, María José de Lancastre y el mismo Tabucchi- acompañados de la jugosa introducción que es «Un baule pieno di gente», a la que siguen una serie de materiales casi de fichero sobre cada uno de los personajes más o menos heteronímicos concebidos por el poeta, así como referencias completas de

44 Ibid., p. 189.

45 Ibid., p. 190.

46 Ibid., p. 192. 
contextualización a las efímeras revistas y corrientes de la modernidad portuguesa; no sólo se incluyen en la antología, por otra parte, poemas del Ortónimo y de los heterónimos más predicados, sino además fragmentos de la prosa narrativa del Livro do Desassossego de Bernardo Soares y los ejercicios de escritura automática de Coelho Pacheco.

Luciana Stegagno Picchio, en su comentario a la obra, ha afirmado que el mérito mayor de Tabucchi es «[...] quello di avere [...] scavalcato, pur senza sottovalutarla, la problematica della frammentazione della personalita $[\ldots]{ }^{47}$ de Pessoa. En efecto, Una sola moltitudine, como acta de defunción de un proceso de mitificación, como definitivo mojón inicial del conocimiento estricto - y tan ancho- del fingidor portugués, es contestación escueta, contundente, a aquellos «[...] lettori innocenti e critici di ogni scuola [i quali girano] con la turcia per ogni angolo dell' [opera di Pessoa] [...] non tanto per sapere che cosa fossero [...] quegli scritti, ma essenzialmente per rispondere all'interrogativo biografico: «Ma chi era, veramente, Fernando Pessoa?»O anche: «Era schizofrenico Fernando Pessoa?» ${ }^{48}$.

La respuesta, paradigmática, la da, en Italia, Tabucchi:

Non cè nessun caso clinico da scoprire nell'eteronimia di Pessoa, solo una «semplice follia», così come forse è «semplice follia» tutta la letteratura. [...] Trovate un uomo cui la vita basti: costui non farà mai letteratura ${ }^{49}$.

47 Luciana S. Picchio, «Pessoa, il poeta è una galassia», Tuttolibri, 12-5-79, pp. 14 s., p. 15.

48 Ibíd., p. 14.

49 Tabucchi, «Un baule pieno di gente», p. 25. Con posterioridad a la fecha marcada como límite del presente trabajo han aparecido Due racconti del mistero ( $A$ very original Dinner del heterónimo Alexander Search y O Roubo da Quinta das Vinhas) en traducción de Amina Di Munno, con prólogo de Tabucchi, Genova-Ivrea, Herodote, 1983; asimismo el volumen colectivo, al cuidado de Tabucchi, Il Poeta e la finzione. Scritti su Fernando Pessoa, Genova, Tilgher, 1983. Fuera del ámbito aquí precisado, además, el artículo de Carlo Vittorio Cattaneo, «Un Poema Blasfemo de Fernando Pessoa», Coloquio / Letras, Lisboa, 50 (1979).

Es de reseñar, por último, un montaje dramático del grupo «Teatroinaria» de Roma, L'altra insonnia, basado en la vida y en la obra del poeta portugués, estrenado en Torino (cf., Osvaldo Guerrieri, «In scena il poeta Pessoa, è uno e centomila», La Stampa, 8-5-86, p. 21). 
